\title{
Vertebral bone marrow edema in a patient with disseminated tuberculosis: a rare case report
}

\begin{abstract}
Bone marrow signal abnormality in the spine and sacrum is a common finding on MRI There are numerous causes of bone marrow signal alteration in spine and sacrum. Inflammatory arthritis, myeloproliferative disorders, radiation and fracture are some of them. We describe a rare case of young immuno-competent male with disseminated tuberculosis involving lungs and sacro-iliac joint having extensive vertebral column high signal intensity.
\end{abstract}

Keywords: sacroilitis, tuberculosis, vertebral bone marrow edema

\author{
Volume 4 Issue I - 2016 \\ Paritosh Garg,' Nikhil Gupta, ${ }^{2}$ Nikhil Nair ${ }^{3}$ \\ 'Department of Pathology, University College of Medical \\ Sciences, India \\ ${ }^{2}$ Department of Rheumatology, University College of Medical \\ Sciences, India \\ ${ }^{3}$ Department of Radio-diagnosis, University of Delhi, India
}

\begin{abstract}
Correspondence: Nikhil Gupta, Department of Rheumatology, University College of Medical Sciences, India,

Email drnikhilguptamamc@gmail.com
\end{abstract}

Received: October 24, 2015 | Published: January 29, 2016

\section{Introduction}

Tuberculosis is a major health problem in developing countries like India. Involvement of the central nervous system (CNS) by the TB accounts for approximately $1 \%$ of all cases of tuberculosis, ${ }^{1}$ half of these involving the spine. Tuberculosis is one of the rare causes of bone marrow signal alteration in vertebral column. Other causes of bone marrow signal alteration in vertebral column includes variants of normal, marrow reconversion, tumour (myeloproliferative disorders, metastatic, or primary), radiation, fracture, degenerative change, infection, inflammatory arthritis, and osteonecrosis. ${ }^{2}$ We describe a rare case of tuberculosis causing extensive signal alteration in vertebral column.

\section{Case description}

A 24year old male presented with complaint of low back pain for 2 months which increased in intensity for last 15 days. The pain was inflammatory, intensified at rest and subsided with motion. Pain was associated with morning stiffness for an hour. There was history of malaise and easy fatigability. There was no history of pain or swelling in his joints, cough, fever, night sweats or weight loss. There was no history of red eye or psoriatic rash. There was no history of tuberculosis in the past. There was no family history of rheumatic disease. On physical examination, his spinal motions were normal and not limited. Modified Schober's test was $4 \mathrm{~cm}$ and chest expansion was $4 \mathrm{~cm}$. There was no tenderness at bilateral sacro-iliac joint.

Patient's complete blood count and peripheral smear was normal. The results of urine culture, blood culture and serology of viruses including EBV, CMV, hepatitis B, C and HIV were negative. Erythrocyte sedimentation rate: $68 \mathrm{~mm} / \mathrm{h}$, C-reactive protein: $24 \mathrm{mg} / \mathrm{L}$ (normal $<5$ ). HLAB27 was negative and mantaux was $14 \mathrm{~mm}$. Blood TB PCR, TB quantiferon Gold test and serology for Brucella was negative. $\mathrm{X}$ rays of the spine, pelvis and sacro-iliac joints were normal. Ophthalmological and skin examination was normal. MRI of the Sacro-iliac joint revealed diffuse patchy marrow edema in the bilateral sacro-iliac joints (Figure 1) involving bilateral sacral ala and juxta articular iliac bones and associated with diffuse patchy marrow edema in the vertebral bodies from thoracic to sacrum vertebrae. There was mild reduction of joint space in the bilateral sacro-iliac joint inferiorly with erosive changes in the articular surface of iliac aspect of right sacro-iliacjoint. MRI of whole spine revealed diffuse patchy marrow edema in the vertebral bodies (Figure 2). Bone marrow examination was essentially normal.

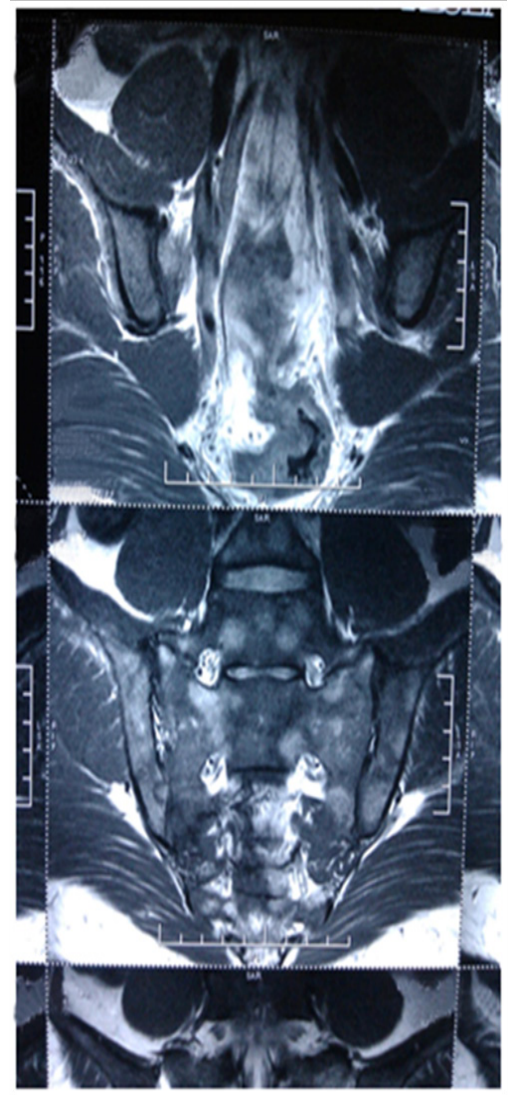

Figure I MRI of the Sacro-iliac joint revealed diffuse patchy marrow edema in the bilateral sacro-iliac joints. 


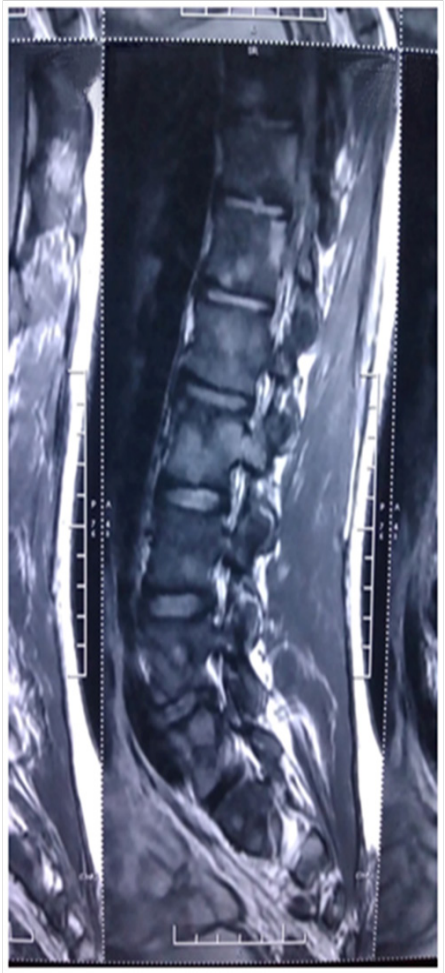

Figure $2 \mathrm{MRI}$ of whole spine revealed diffuse patchy marrow edema in the vertebral bodies.

CECT chest and abdomen revealed nodular infiltrates with fibrotic bands in apical segment of right upper lobe with few rim enhancing necrotic lymph nodes at pre-tracheal, right para-tracheal, pre carinal and sub carinal region. Bone marrow examination was done which was normal. Lymph node biopsy of pre-tracheal nodes was done which showed caseous necrosis and langhans type giant cells (Figure $3)$.

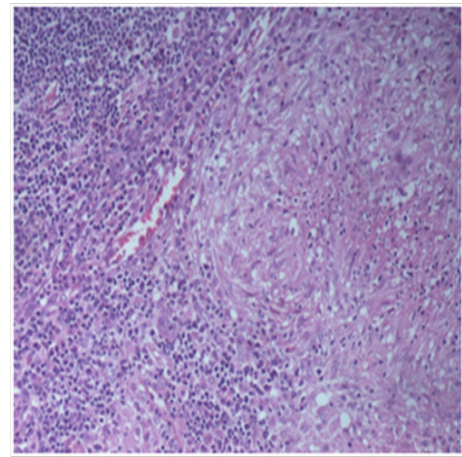

Figure 3 Lymph node biopsy of pre-tracheal nodes was done which showed caseous necrosis and langhans type giant cells.

A diagnosis of disseminated tubercular was made and patient was started on anti tubercular drugs (Isoniazid, rifampicin, ethambutal and pyrazinamide). Patient reported improvement in his symptoms after 1 month of treatment. Finally, the symptoms subsided and the patient was relieved of his ailment completely. Now, after 1 year course of anti tubercular drug therapy the patient is absolutely normal.

\section{Discussion}

Tuberculosis (TB) is a common disease in Asian countries Tuberculosis can affect almost every organ of the body.
Musculoskeletal involvement in TB is uncommon, occurring in 1-3\% of cases. ${ }^{3}$ In patients of skeletal TB, involvement of sacroiliac joint (SIJ) had been reported in 3-9.7\% of patients. ${ }^{4}$ TB sacro-ilitis presents with vague symptoms of low back ache, fatigue and stiffness. Thus due to its non specific clinical features, it is often missed or diagnosed late. TB sacro-ilitis is a devastating disease leading to high morbidity and thus needing early diagnosis and treatment. ${ }^{5}$

Tuberculosis is a rare cause of bone marrow signal alteration in vertebral column. Causes of diffuse bone marrow signal alteration in vertebral column by includes variants of normal, marrow reconversion and myeloproliferative disorders as luekemia, multiple myeloma, polycythemia vera and myelofibrosis. Causes of focal bone marrow edema include solitary metastatic lesions, lymphoma or primary tumors as chordoma. Radiation, fracture, degenerative change, infection, inflammatory arthritis, and osteonecrosis are some of the other causes of focal bone marrow edema. MRI-STIR and T2 fast spin-echo-weighted fat-suppression images are extremely sensitive for detecting fluid and show bone marrow edema as hyper-intensities. MRI appearance of infection in the sacrum and vertebral spine occurs as focal or multifocal marrow signal abnormalities that are most often cantered around an intervertebral disk or the sacroiliac joints. ${ }^{2}$

Spinal tuberculosis is thought to originate in the vertebral body. It spreads beneath the longitudinal ligaments to involve adjacent vertebral bodies without affecting the neighbouring disks. Other characteristics of tuberculosis include skip lesions and involvement of multiple vertebral bodies or only a portion of a vertebral body (such as the posterior elements). ${ }^{2}$ However, these MRI features can make it difficult to differentiate tuberculosis infection from such neoplasm as lymphoma or metastatic disease.

Definitive diagnosis requires fine needle aspiration or open biopsy. The presence of AFB in direct smears and stains or the growth of the bacilli in the LJ culture or the granulomatous lesion in the histologic specimen will confirm the diagnosis of tuberculosis. Anti-tubercles chemotherapy agents are used in the treatment of tubercular sacroilitis. Our patient had constitutional symptoms of malaise and fatigue. He did not have any other symptom as bowel complaints, uveitis or psoriasis to support the diagnosis of spondyloarthropathy. Moreover, he was found to be negative for HLAB27. His Chest CT along with lymph node biopsy had a supportive evidence of tuberculosis. Our patient responded very well to the anti tubercular drugs and became normal later on.

\section{Conclusion}

Tuberculosis can present with sacro-ilitis and vertebral bone marrow edema. A high suspicion should be kept as the disease is associated with high morbidity. Non specific symptoms being its presentation, a high suspicion should be kept. Early treatment may prevent further complications of TB. Vertebral bone marrow edema may be seen in tuberculosis. Other etiologies such as tumour (myeloproliferative disorders, metastatic, or primary), radiation, fracture, degenerative change, infection, inflammatory arthritis, and osteonecrosis must be ruled.

\section{Acknowledgements}

None.

\section{Conflict of interest}

The author declares no conflict of interest. 


\section{References}

1. Jain AK, Dhammi IK. Tuberculosis of the spine: A review. Clin Orthop Relat Res. 2007;460:39-49.

2. Long SS, Yablon CM, Eisenberg RL. Bone marrow signal alteration in the spine and sacrum. AJR Am J Roentgenol. 2010;195(3):178-200.

3. Tuli SM. General principles of osteoarticular tuberculosis. Clin Orthop Relat Res. 2002;398:11-19.
4. Pouchot J, Vinceneux P, Barge J, et al. Tuberculosis of the sacroiliac joint: clinical features, outcome, and evaluation of closed needle biopsy in 11 consecutive cases. Am J Med. 1998;84(3):622-628.

5. Papadopoulos ECh, Papagelopoulos PJ, Savvidou OD, et al. Tuberculous sacroiliitis. Orthopedics. 2003;26(6):653-657.

6. Hendrix RW, Lin PJ, Kane WJ. Simplified aspiration or injection technique for the sacro-iliac joint. J Bone Joint Surg Am. 1982;64(8):12491252 